Check for updates

Cite this: RSC Adv., 2017, 7, 42468

\title{
Synthesis, properties and gas separation performance of poly(arylene ether sulfone) containing imide pendant groups $\uparrow$
}

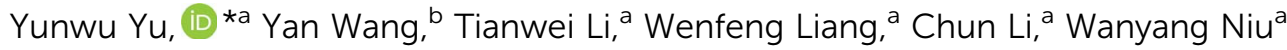 \\ and Lili Gao ${ }^{a}$
}

The precursor poly(arylene ether sulfone) containing amino-groups was synthesized by a nucleophilic polymerization route, and then modified using three commercially available anhydrides. The anhydride modified polymers had fractional free volume (FFV) values in the range of 0.128-0.162, and good mechanical, thermal and gas transport properties, which were correlated with the polymer chain structure. They showed higher glass transition temperatures in the range $207-227^{\circ} \mathrm{C}$ compared with the precursor polymers due to the introduction of imide pendant groups, and better thermal stability with $5 \%$ weight loss above $512{ }^{\circ} \mathrm{C}$. The incorporation of imide pendant groups into poly(arylene ether sulfone)s improved the gas transport performance evidently due to the higher fractional free volume. The anhydride modified polymer Br-PAES-20 possesses higher permeability and permselectivity simultaneously. The most permeable membrane PEP-PAES- 60 showed permeability coefficients of 8.66 barrers to $\mathrm{O}_{2}, 0.64$ barrers to $\mathrm{N}_{2}$ and 13.56 barrers to $\mathrm{CO}_{2}$, with an ideal selectivity factor of 13.53 for the $\mathrm{O}_{2} / \mathrm{N}_{2}$ pair.

Received 4th July 2017

Accepted 27th August 2017

DOI: 10.1039/c7ra07355k

rsc.li/rsc-advances

\section{Introduction}

Membrane-based separation technology has become promising in both cost and performance in the field of gas separation, and is even considered as a separation technology that could change the world. ${ }^{1}$ Polymeric membranes have been of great interest in the separation of many important industrial gases, such as oxygen/nitrogen enriched, natural gas dehydration, hydrogen recovery from the purge steam in ammonia plants, and light olefin-paraffin separation. ${ }^{2,3}$ Such interest in polymeric membrane technology is due to the easy processability of membranes and low capital investment cost.

Gas separation based polymeric membranes seems to possess tremendous potential as an energy-efficient alternative for room-temperature separations. To push forward the predominance of membrane-based separation technology, polymeric membranes should possess both higher permeability and selectivity. ${ }^{4,5}$ However, there is a trade-off relation between permeability and selectivity of polymeric membranes. ${ }^{6,7}$ The upper bound line to the trade-off of gas permeability versus gas selectivity was obtained by Robeson ${ }^{6}$ in 1991 for several gas

${ }^{a}$ School of Materials Science and Engineering, Shenyang Jianzhu University, Shenyang 110168, P.R. China. E-mail: diabloyyw@sina.com

${ }^{b}$ Alan G MacDiarmid Institute, Jilin University, Changchun 130012, P.R. China

$\dagger$ Electronic supplementary information (ESI) available. See DOI: 10.1039/c7ra07355k pairs. As the separation performance of polymers improving, a higher upper bound line was established in $2008 .^{7}$

The transport of gas molecules through dense polymeric membranes is usually described by the solution-diffusion mechanism. ${ }^{8}$ In this mechanism, the permeants dissolve in the polymeric membrane and then diffuse through the membrane down a concentration gradient. The permeation process could be viewed as a product of solution and diffusion. Therefore the permeability coefficient could be described by the following equation: ${ }^{9}$

$$
P=D \times S
$$

where $P$ is the permeability coefficient, $D$ is the diffusion coefficient, and $S$ is the sorption coefficient.

According to solution-diffusion mechanism, a very promising way to develop new polymeric materials with improved properties consists of optimizing the structure of the main chains and pendant groups to enhance fractional free volume domains with a tailored distribution of pore sizes in order to increase the diffusivity and also with restrictive or selective channels communicating the interchain voids to increase the permselectivity. ${ }^{\mathbf{1 0}}$ Glassy polymers that have lower intrasegmental mobility and longer relaxation time are preferentially chosen as practical membrane materials, amongst the various polymer materials, for almost all industrial gas separation because of their inherent high selectivity, excellent thermal and chemical stability, and good mechanical properties. ${ }^{11}$ 
In general, glassy polymers are characterized by the relatively small fraction of free volume, so the more permeable species in glassy polymers are those with low kinetic molecular diameter and selectivity is due to the difference in kinetic molecular dimension. The stiff structure of aromatic polymers, such as poly(aryl ether ketone), polysulfone, polyphenylene sulfide, polyimide etc., reduces the intrasegmental mobility and limits the degree of chain packing which in turn increases the free volume. Therefore, aromatic glassy polymers could possess relatively higher diffusivity. In particular, aromatic polyimides are extensive used for their excellent thermal oxidative stability, exceptional mechanical properties, along with an extraordinary ability to separate complex mixtures of gases in diverse applications. ${ }^{\mathbf{1 2 - 1 4}}$

On the basis of facts above, we chose poly(arylene ether sulfone) contained amino-groups as precursor, and then introduced imide pendant groups by the reaction between amine-groups and anhydrides. Through this approach, we got a series of newly modified poly(arylene ether sulfone). The variation in chemical structure of polymers was expected to result in interesting variation in physical properties, for instance, packing density and segmental motion of the polymer chain, and thus should promote the gas permeation in membranes. The effect of structural variation on the gas separation properties has been investigated through the calculation of fractional free volume and gas permeation measurement. Moreover, thermally stable imide groups could improve heat resistance of polymers.

\section{Experimental}

\section{A Materials}

4,4'-Difluorodiphenylsulfone (DFDPS), 4,4'-(hexafluoroisopropylidene)diphenol, 4-nitroaniline, 1,4-benzoquinone were purchased from Aldrich. Three kinds of acid anhydride, 4-chlorophthalic anhydride, 4-bromo-1,8-naphthalic anhydride and 4-phenylethynylphthalic anhydride, were purchased from Tokyo Chemical Industry. $\mathrm{K}_{2} \mathrm{CO}_{3}$ purchased from Sinopharm Chemical Reagent Co., Ltd was ground into fine powder and dried at $120^{\circ} \mathrm{C}$ for $24 \mathrm{~h}$ before polymerization. All the organic solvents were purchased from Sinopharm Chemical Reagent Co., Ltd and purified by conventional methods.

\section{B Synthesis of Am-PAES}

The bisphenol monomer containing amino-group was 4-aminophenyl hydroquinone (4-AmPHQ), synthesized in our lab through a two-step diazonium salt coupling-reduction reaction shown in Scheme $1 .{ }^{15}$ A series of precursor polymers with

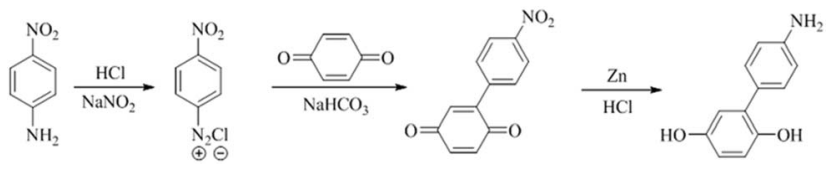

Scheme 1 Synthesis of (4-amino)phenyl hydroquinone. different contents of amino-group (20\%, 40\%) were prepared through the route shown in Scheme 2 according to the reported procedures previously. ${ }^{16}$

A typical synthesis procedure of Am-PAES-20 (4-AmPHQ mole percent content was $20 \%$ ) as follows: $0.4024 \mathrm{~g}$ (2 mmol) 4 AmPHQ, $2.6898 \mathrm{~g}$ (8 $\mathrm{mmol}$ ) 4,4'-(hexafluoroisopropylidene) diphenol, $2.5425 \mathrm{~g}$ DFDPS (10 mmol) and $1.518 \mathrm{~g} \mathrm{~K}_{2} \mathrm{CO}_{3}$ were added into three-necked flask with a Dean-Stark trap, condenser, mechanical stirrer and nitrogen inlet \& outlet. $\mathrm{N}$ Methyl-2-pyrrolidone $(20 \mathrm{~mL})$ and toluene $(20 \mathrm{~mL})$ were used as solvent and azeotropic reagent, respectively. The reaction mixture was refluxed at $130^{\circ} \mathrm{C}$ for $3 \mathrm{~h}$ to dehydrate the system completely. After dehydration and removal of toluene, the reaction temperature was raised to $180{ }^{\circ} \mathrm{C}$ gradually and held at this temperature for $3 \mathrm{~h}$. Then the temperature was raised to $200{ }^{\circ} \mathrm{C}$, and held at this temperature till the viscosity did not increase any further. A viscous solution was obtained and then poured into the mixture of ice and water slowly to precipitate the polymer. The polymer was pulverized into a powder using a high-speed disintegrator. The polymer powder was washed several times with ethanol and hot water with a nitrogen inlet. Finally the light yellow polymer powder was dried in a vacuum oven at $100{ }^{\circ} \mathrm{C}$. Another precursor polymer Am-PAES-60 was prepared following the similar procedure.

\section{Synthesis of anhydride modified PAES}

In this work, the modification of Am-PAES derived from ringopening addition reaction with anhydrides, including 4-chlorophthalic anhydride, 4-bromo-1,8-naphthalic anhydride and 4phenylethynylphthalic anhydride, as shown in Scheme 3. In a typical experiment, Cl-PAES-20 was prepared as follows: AmPAES-20 (2 g) and 4-chlorophthalic anhydride (the mole ratio of anhydride and amino-group in PAES was $5: 1$ ) were dissolved in $20 \mathrm{~mL}$ NMP. The mixture was magnetically stirred for $12 \mathrm{~h}$ at $80{ }^{\circ} \mathrm{C}$ under anaerobic condition to yield a solution of PAES containing amic acid pendants. Chemical imidization was carried out via the addition of $2 \mathrm{~mL}$ mixture of acetic anhydride/ pyridine $(2 / 1 \mathrm{v} / \mathrm{v})$ into the solution with mechanical stirring, and then stirred at $80{ }^{\circ} \mathrm{C}$ for $4 \mathrm{~h}$. The solution finally obtained was poured into ethanol to get a precipitate, the imide group modified PAES. Cl-PAES-20 powder was collected by filtration,

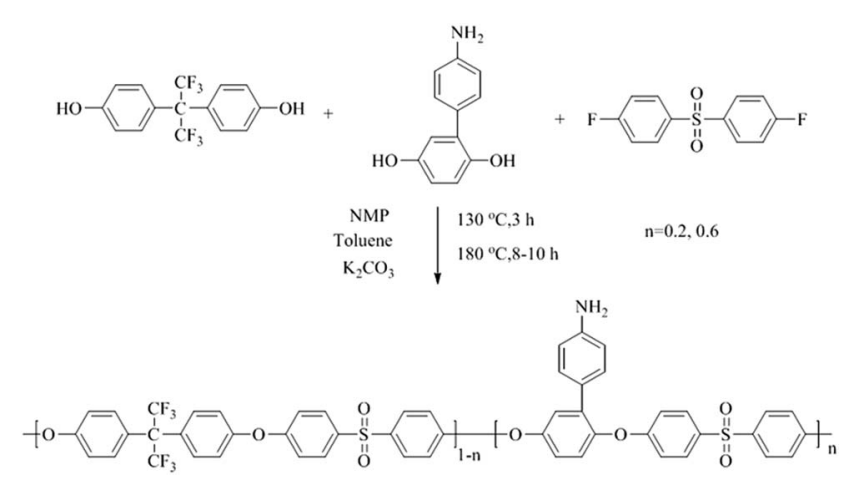

Scheme 2 Synthesis route of poly(arylene ether sulfone) with 4amino-phenyl pendant group. 

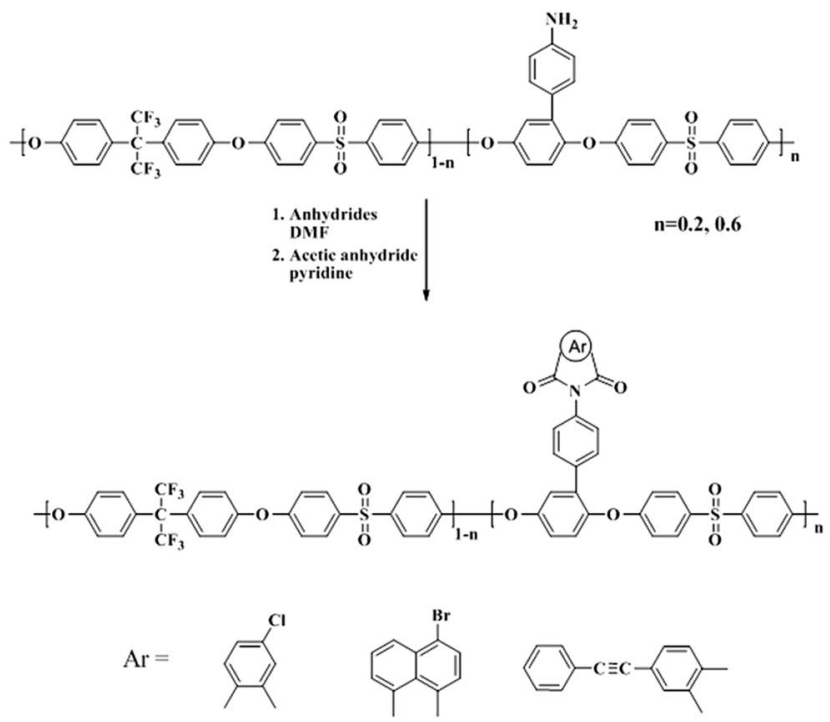

Scheme 3 Synthesis route of anhydride modified PAES.

washed thoroughly by acetone extraction, and dried at $80{ }^{\circ} \mathrm{C}$ in vacuum. Other anhydride modified polymers were synthesized in an analogous procedure.

\section{Membrane preparation}

All Am-PAES membranes and anhydride modified PAES membranes were prepared by solution casting. Each polymer sample ( $1 \mathrm{~g})$ was dissolved into NMP $(12 \mathrm{~mL})$ to form a solution. After being filtered, solutions were poured onto clean glass plates and kept in an oven initially at $60{ }^{\circ} \mathrm{C}$ overnight to remove solvent slowly, then at $80{ }^{\circ} \mathrm{C}$ and $120^{\circ} \mathrm{C}$ successively for several hours to remove residual solvent completely. The thickness of membranes we got was in the range of 85-95 $\mu \mathrm{m}$. Part of PEPPAES membranes were treated at $390{ }^{\circ} \mathrm{C}$ for $2 \mathrm{~h}$ under $\mathrm{N}_{2}$ atmosphere, named as TPEP-PAES.

\section{E Characterization and measurement}

${ }^{1} \mathrm{H}-\mathrm{NMR}$ spectra were carried out on a Bruker 510 spectrometer $\left(500 \mathrm{MHz}\right.$ for $\left.{ }^{1} \mathrm{H}\right)$ using DMSO- $d_{6}$ as solvent. FTIR spectra were recorded via the $\mathrm{KBr}$ pellet method by using a Nicolet iS5 FTIR spectrophotometer. The $T_{\mathrm{g}} \mathrm{S}$ were determined with DSC (Mettler Toledo DSC 821e instrument) at a heating rate of $20{ }^{\circ} \mathrm{C} \mathrm{min}{ }^{-1}$ under nitrogen atmosphere. Thermo gravimetric analysis (TGA) was employed to assess thermal stability of polymers with a Netzch Sta 449c thermal analyzer system. Before analysis, the polymer powders were dried and kept in the TGA furnace at $120{ }^{\circ} \mathrm{C}$ in nitrogen atmosphere for $15 \mathrm{~min}$. The samples were cooled to $100{ }^{\circ} \mathrm{C}$ and then reheated to $800{ }^{\circ} \mathrm{C}$ at a rate of $10{ }^{\circ} \mathrm{C} \min ^{-1}$ in nitrogen atmosphere, and the temperatures at $5 \%$ and $10 \%$ weight loss were recorded for each sample.

The mechanical properties of membranes were measured at room temperature on SHIMADIU AG-I $1 \mathrm{KN}$ at a strain rate of $2 \mathrm{~mm} \min ^{-1}$. The size of samples was $20 \mathrm{~mm} \times 4 \mathrm{~mm}$. The samples were dried at $120{ }^{\circ} \mathrm{C}$ in vacuum oven for $24 \mathrm{~h}$ before measurement.
Single gas permeability coefficients $(P)$ of the pure gases He, $\mathrm{N}_{2}, \mathrm{O}_{2}$ and $\mathrm{CO}_{2}$ were measured on polymer dense membranes by the constant volume method at $25{ }^{\circ} \mathrm{C}$ with an upstream pressure of 1 atm. ${ }^{17,18}$ The permeability of each pure gas was calculated by the following equation from the flux of pure gas on the permeating side.

$$
P=\frac{\text { Flux }}{A \Delta p / l}
$$

where $\Delta p, A$ and $l$ are the partial pressure difference of the gases transmembrane, effective permeating area and the thickness of the tested membrane respectively.

The ideal permselectivities for various gas pairs were calculated based on the permeation rate of pure gases.

$$
\alpha_{\mathrm{AB}}=\frac{P_{\mathrm{A}}}{P_{\mathrm{B}}}
$$

where $P_{\mathrm{A}}$ and $P_{\mathrm{B}}$ are the permeation rate for gas $\mathrm{A}$ and $\mathrm{B}$, respectively. For getting good reproducibility, the replicate experiments for each membrane were performed with three different samples prepared at the same time and the data of gas permeation appeared in this paper were the average results.

The effective diffusion coefficient $D$ that is an apparent one for the glassy polymers ${ }^{\mathbf{1 9}}$ is calculated from the time-lag $\theta$ according to the following equation:

$$
D=\frac{d^{2}}{6 \theta}
$$

\section{F Fractional free volume (FFV) measurement}

The fractional free volume (FFV) was estimated using experimental density $\rho$ and van der Waals volumes $V_{\mathrm{w}}$ of the atoms forming the repeat units. According to the Bondi method, ${ }^{20}$ fractional free volume is calculated using the following equations:

$$
\begin{gathered}
\mathrm{FFV}=V_{\mathrm{f}} / V_{\mathrm{sp}} \\
V_{\mathrm{f}}=V_{\mathrm{sp}}-1.3 V_{\mathrm{w}}
\end{gathered}
$$

where $V_{\mathrm{f}}\left(\mathrm{cm}^{3} \mathrm{~g}^{-1}\right)$ is the free volume of a polymer, the specific volume $V_{\text {sp }}$ is defined as reciprocal polymer density $(1 / \rho)$, the van der Waals volume $V_{\mathrm{w}}$ can be calculated using a group contribution method, and the universal "packing coefficient" estimated by Bondi equal to 1.3 is used to convert the van der Waals volume of the repeat unit in the occupied volume.

The correctness of this approach for all the polymers and in relation to transport of various gases has been discussed. ${ }^{19}$ It can be assumed that a large scatter of the empirical correlations of diffusion and permeability coefficients with $V_{\mathrm{f}}$ and FFV often observed for various polymers can be induced by the only approximate validity of these equations.

The polymer density $\rho$ is the density of the film $\left(\mathrm{g} \mathrm{cm}^{-3}\right)$, which is determined by buoyancy method on a SD-200L electronic densimeter from Alfa Mirage Co., Ltd, Japan, and the density was calculated as follows: 


$$
\rho=\frac{w_{0}}{w_{0}-w_{1}} \rho_{\mathrm{H}_{2} \mathrm{O}}
$$

where $w_{0}$ and $w_{1}$ are the film weights in air and water respectively.

\section{Results and discussion}

\section{A Synthesis and characterization of polymers}

The method of synthesizing precursor polymer Am-PAES has been described in detail in previous study. The modification reaction route was similar to the conventional two-step procedure for the synthesis of polyimides of ring-opening addition at $80{ }^{\circ} \mathrm{C}$ to form amide acid pendants and chemical imidization by adding mixture of acetic anhydride/pyridine.

The structures of the previous polymer and anhydride modified polymers have been confirmed by FT-IR and ${ }^{1} \mathrm{H}-\mathrm{NMR}$ spectra. Fig. 1 shows the FT-IR spectra of anhydride modified polymers, Cl-PAES-60, Br-PAES-60 and PEP-PAES-60. The FT-IR spectra exhibited characteristic imide group absorptions peaks at around 1784 and $1730 \mathrm{~cm}^{-1}$ (typical absorptions of imide carbonyl asymmetrical and symmetrical stretchings) and did not show the amide carbonyl peak at $1650 \mathrm{~cm}^{-1}$, thus demonstrating complete imidization during modification. $1374 \mathrm{~cm}^{-1}$ (C-N-C stretching), 1093 and $730 \mathrm{~cm}^{-1}$ (imide ring deformation), $1279-1190 \mathrm{~cm}^{-1}$ (C-F and $\mathrm{Ph}-\mathrm{O}-\mathrm{Ph}$ stretchings) absorption bands are also clearly visible. The FT-IR spectrum of PEP-PAES-60 exhibited characteristic acetylene group stretching absorptions at $2215 \mathrm{~cm}^{-1} \cdot{ }^{1} \mathrm{H}-\mathrm{NMR}$ spectra of Cl-PAES, Br-PAES and PEP-PAES are shown in Fig. 2. All protons resonate in the region of 6.9-8.1 ppm and have been well assigned. In all spectra, the protons $\mathrm{H}_{7}$ and $\mathrm{H}_{8}$ shifted to a lower field compared with the same protons of Am-PEAS due to the electronwithdrawing property of imide group. As shown in complete spectra provided in the ESI, $\dagger$ no resonate of amino protons (around $5.2 \mathrm{ppm}$ ) were observed after modification. The above results demonstrate that these three anhydrides hold a good reaction activity with amino-groups in Am-PEAS. Fully chemical

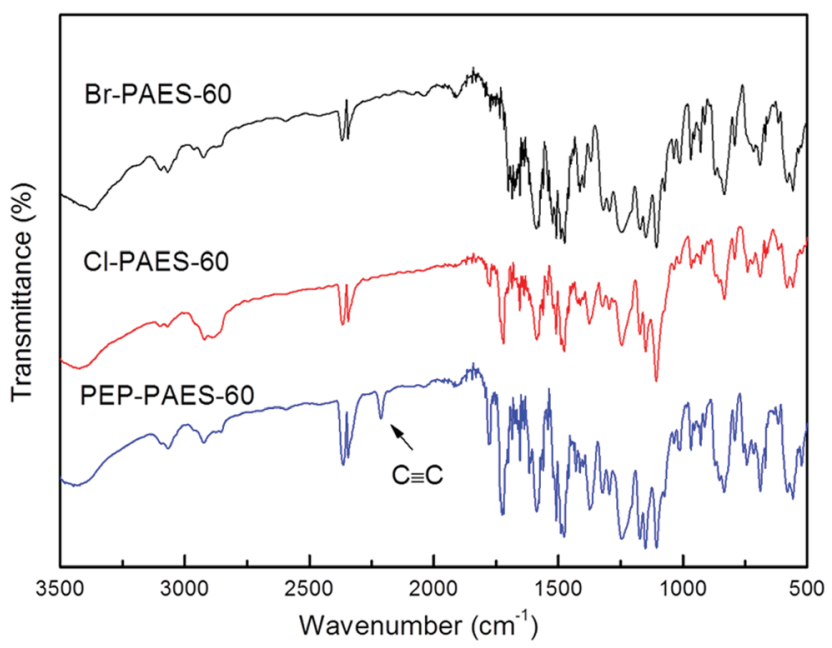

Fig. 1 FT-IR spectra of anhydride modified PAESs.
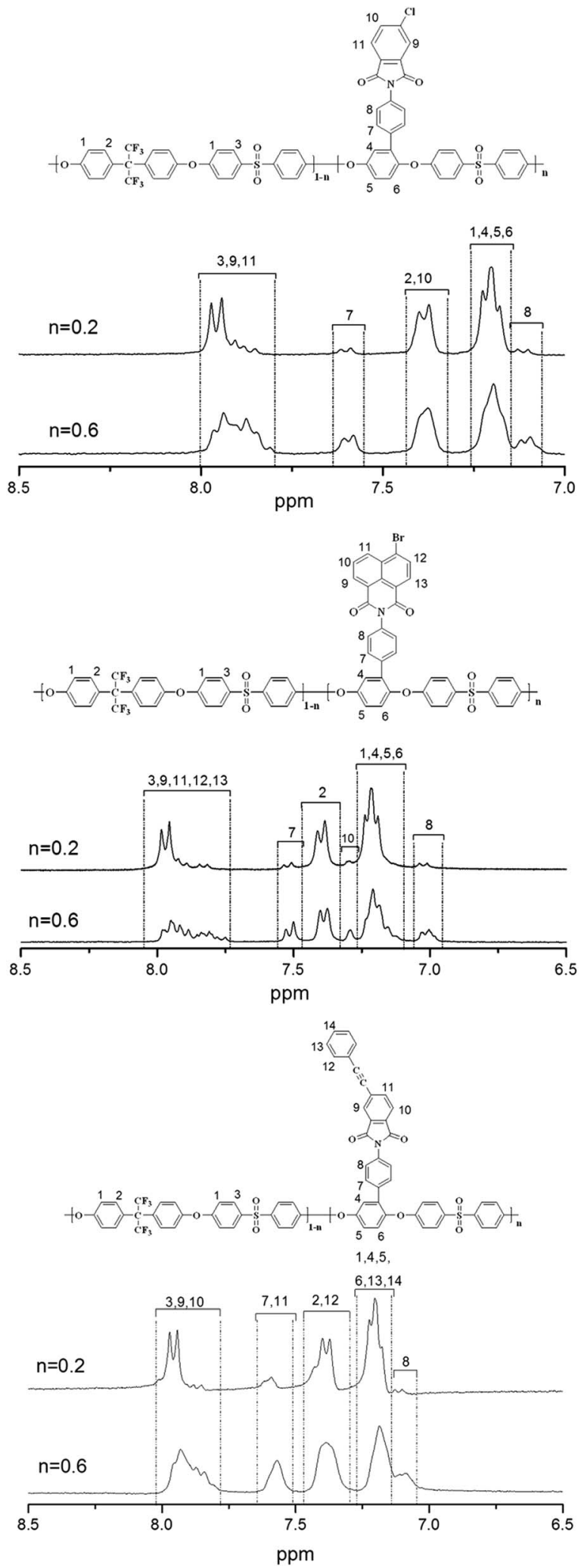

Fig. $2{ }^{1} \mathrm{H}-\mathrm{NMR}$ spectra of anhydride modified PAESs.

imidization catalyzed by acetic anhydride and pyridine could be achieved at $80^{\circ} \mathrm{C}$.

For synthesis of polyimides, the anhydride monomer with an electron withdrawing substituent and the diamine monomer 
with an electron donating substituent on the aromatic ring have higher reactivity. ${ }^{21}$ The three anhydrides we chose in this study had enough reaction activity with amino-groups in Am-PEAS due to electron withdrawing $-\mathrm{Cl},-\mathrm{Br}$ or conjugated phenylethynyl group. In actual synthesis of poly amic acid, compared with aromatic dianhydride monomer, the aromatic diamine monomer has a greater influence on the formation of amide acid.

\section{B Polymer solubility}

The solubility of the polymers was tested in various solvents, and the results were summarized in Table 1 . The aminated poly(arylene ether sulfone)s and anhydride modified poly(arylene ether sulfone)s exhibited good solubility in common organic solvents. It was attempted to obtain 10\% (weight/ volume) solution of all polymers in several organic solvents. These polymers were soluble in chloroform $\left(\mathrm{CHCl}_{3}\right)$, dichloromethane $\left(\mathrm{CH}_{2} \mathrm{Cl}_{2}\right)$, and in dipolar aprotic solvents $N, N^{\prime}$-dimethylformamide (DMF), N, $N^{\prime}$-dimethylacetamide (DMAc), $N$ methyl pyrrolidone (NMP), dimethyl sulfoxide (DMSO) at room temperature. The anhydride modified polymers showed relatively poor solubility in tetrahydrofuran (THF) at room temperature, needed more stirring and solvent to dissolve completely. All the polymers were insoluble in acetone. The good solubility could be due to the presence of hexafluoropropane $-\mathrm{C}\left(\mathrm{CF}_{3}\right)_{2}-$ in polymer main chains. The $-\mathrm{Cl}$ and -Br in imide moiety also made a contribution to the solubility. The thermally treated PEP-PAES samples exhibited good solvent resistance. They were insoluble in the solvents previously mentioned, even exhibited good short-term swelling resistance.

\section{Thermal properties of polymers}

The thermal behavior data of all the polymers are listed in Table 2. Generally, the order of $T_{\mathrm{g}}$ correlates with both molecular packing and chain conformation (chain rigidity and interaction) of polymers. Precursor polymers showed higher $T_{\mathrm{g}}$ due to the strongly hydrogen bond interaction between chains caused by $-\mathrm{NH}_{2}$ groups. The DSC curves of anhydride modified PAESs are shown in Fig. 3. The $T_{\mathrm{g}}$ values of Am-PAESs and anhydride modified PAESs were, respectively, in ranges of 205$209{ }^{\circ} \mathrm{C}$ and $207-227{ }^{\circ} \mathrm{C}$, which increased with the amount of imide group. As we expected, the $T_{\mathrm{g}}$ values of modified PAESs increased with the increasing rigidity of PAES backbones

Table 1 Solubility of polymers ${ }^{a}$

\begin{tabular}{llllllll}
\hline Polymers & NMP & DMF & DMAc & DMSO & $\mathrm{CHCl}_{3}$ & THF & Acetone \\
\hline Am-PAES & ++ & ++ & ++ & + & + & + & - \\
Cl-PEAS & ++ & ++ & ++ & ++ & ++ & + & - \\
Br-PAES & ++ & ++ & ++ & ++ & ++ & + & - \\
PEP-PAES & ++ & ++ & ++ & + & + & + & - \\
TPEP-PAES & - & - & - & - & - & - & -
\end{tabular}

${ }^{a}++$, soluble at room temperature; + , soluble with more stirring or heating; - , insoluble at room temperature.
Table 2 Thermal properties of precursor polymers and anhydride modified polymers

\begin{tabular}{lllll}
\hline Polymer & $T_{\mathrm{g}}\left({ }^{\circ} \mathrm{C}\right)$ & $T_{\mathrm{d} 5 \%}\left({ }^{\circ} \mathrm{C}\right)$ & $T_{\mathrm{d} 10 \%}\left({ }^{\circ} \mathrm{C}\right)$ & Char yield (\%) \\
\hline Am-PAES-20 & 205 & 500 & 518 & 52 \\
Am-PAES-60 & 209 & 463 & 490 & 53 \\
Cl-PAES-20 & 207 & 512 & 535 & 70 \\
Cl-PAES-60 & 226 & 530 & 548 & 62 \\
Br-PAES-20 & 210 & 513 & 530 & 55 \\
Br-PAES-60 & 227 & 525 & 541 & 44 \\
PEP-PAES-20 & 208 & 512 & 535 & 49 \\
PEP-PAES-60 & 221 & 521 & 543 & 57 \\
\end{tabular}

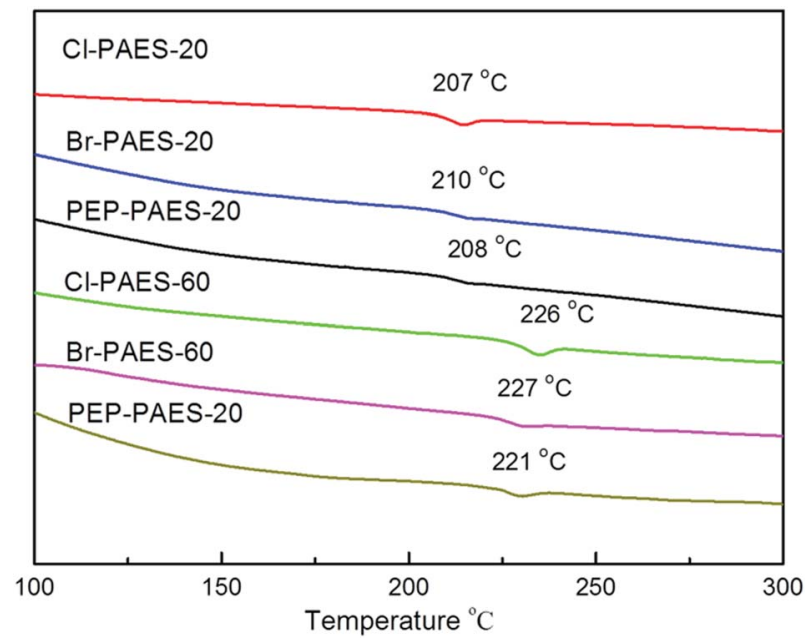

Fig. 3 DSC curves of anhydride modified PAESs.

attributed to the introduction of bulky pendant imide groups which having large volume and high rigidity and restricting polymer chain segment motion. PAESs modified by 4-bromo1,8-naphthalic anhydride have maximum $\Delta T_{\mathrm{g}} \mathrm{s}\left(5{ }^{\circ} \mathrm{C}\right.$ and 18 $\left.{ }^{\circ} \mathrm{C}\right)$ that benefited from the fused ring structure of naphthyl group. The DSC curve of PEP-PAES- 60 from $100{ }^{\circ} \mathrm{C}$ to $500{ }^{\circ} \mathrm{C}$ was shown in Fig. 4, in which an exothermal peak ranged from $370{ }^{\circ} \mathrm{C}$ to $450{ }^{\circ} \mathrm{C}$ could be observed. The exotherm could not be detected in the curves of PAESs modified by other anhydride. It could be attributed to the crosslinking reaction of phenylethynyl moieties in PAESs modified by 4-phenylethynylphthalic anhydride (Fig. 5). ${ }^{22}$

Thermal stabilities of the PAESs evaluated by TGA are also listed in Table 2. $T_{\mathrm{d}} \mathrm{S}$ of Am-PAESs increases with increasing the content of $-\mathrm{C}\left(\mathrm{CF}_{3}\right)_{2}$ - component due to the presence of stronger $\mathrm{C}-\mathrm{F}$ bonds with higher thermal stability ${ }^{\mathbf{2 3 , 2 4}}$ than $-\mathrm{NH}_{2}$. Besides, $-\mathrm{C}\left(\mathrm{CF}_{3}\right)_{2}-$ is in the backbone and has a more significant contribution to thermal stability. Compared with Am-PAESs, all anhydride modified PAESs exhibited more excellent thermal stability, and no obvious decomposition was observed below $500{ }^{\circ} \mathrm{C}$. Compared with precursor polymers, anhydride modified PAESs showed an opposite trend of the $T_{\mathrm{d}}$ with the pendant content. This was because that the more amino groups in the precursor polymer, the more imide pendant groups with 


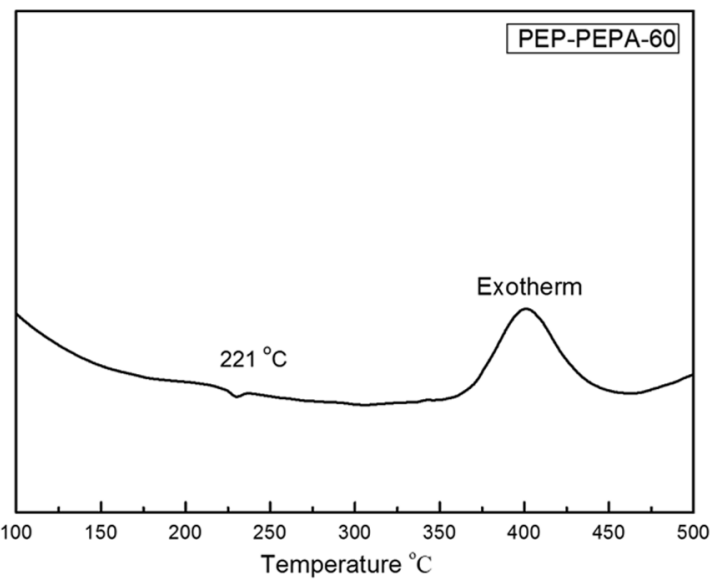

Fig. 4 The DSC curve of PEP-PAES-60 in temperature range $100{ }^{\circ} \mathrm{C}$ to $500^{\circ} \mathrm{C}$.

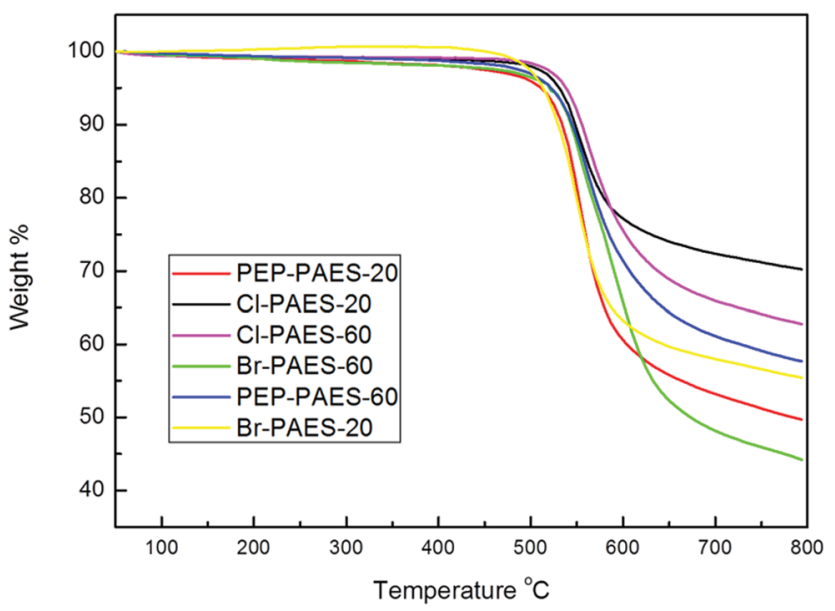

Fig. 5 TGA curves of anhydride modified polymers in $\mathrm{N}_{2}$.

excellent thermal stability were introduced (the maximum $\Delta T_{\mathrm{d} 5 \%}$ and $\Delta T_{\mathrm{d} 10 \%}$ were $67^{\circ} \mathrm{C}$ and $58^{\circ} \mathrm{C}$, in the case of Cl-PAES60). Their char yield at $800^{\circ} \mathrm{C}$ in nitrogen was in the range of 44$70 \mathrm{wt} \%$. The TGA data indicated that these PAESs had fairly higher thermal stability regardless of the introduction of the imide pendant groups. For Cl-PAES and Br-PAES, the char yield was decreased with the increase of the imide group content while an opposite trend occurs for PEP-PAES. We postulated that the marked difference in char yield was mainly due to crosslinking of ethynyl groups during temperature rising.

\section{Mechanical properties}

The mechanical properties of PAES membranes are summarized in Table 3. The PAES membranes had tensile strength of 69-110 MPa, tensile modulus of 2.5-5.2 GPa, and maximum elongation of $2.8-10.8 \%$. The strength of polymers decreased with the introducing of imide pendant groups. We thought it was because that the introduction of bulky imide substituent reduced the bulk density and entanglement degree of polymer chains, led to the lower strength of membranes. ${ }^{25}$ TPEP-PAES showed much higher tensile strength and modulus, meanwhile, a lower elongation at break as a result of ethynyl groups' crosslinking. Anyway, all of the membranes possess high enough toughness for gas permeation test.

\section{E Gas permeability and permselectivity}

It is well-known that permeation of gas molecules in dense polymer membranes following the solution-diffusion mechanism, ${ }^{26}$ and then the permeability coefficient $(P)$ is determined by the solubility coefficient $(S)$ and the diffusion coefficient $(D)$, $P=D S$. The diffusion coefficient of common gases, which have no obvious interactions with polymers, is considered as a function of the effective molecular diameter of the gas molecule ${ }^{6}$ and the diffusivity coefficient correlates very well with the FFV of polymers. ${ }^{27}$ In this condition, the $P$ is mainly determined by the diffusion coefficient. The mean permeability coefficients of four different gases $\mathrm{O}_{2}, \mathrm{~N}_{2}, \mathrm{CO}_{2}$ and $\mathrm{He}$ through these PAES membranes and ideal permselectivity values for different gas pairs are presented in Table 4 . To explain the effect of imide pendant groups on gas permeation of polymers, the fraction free volume (FFV) of polymers was calculated by utilizing the Bondi's group contribution method and listed in Table 4.

For all the PAES membranes, the gas permeability coefficient decreases following the sequence $P(\mathrm{He})>P\left(\mathrm{CO}_{2}\right)>P\left(\mathrm{O}_{2}\right)>P\left(\mathrm{~N}_{2}\right)$, which is in the reversed order of their respective kinetic diameter, $\mathrm{He}(2.60 \AA)<\mathrm{CO}_{2}(3.3 \AA)<\mathrm{O}_{2}(3.46 \AA)<\mathrm{N}_{2}(3.64 \AA) .{ }^{11}$ Compared with anhydride modified PAESs, precursor polymers Am-PAES-20 and Am-PAES-60 exhibited lower $\mathrm{O}_{2}, \mathrm{~N}_{2}, \mathrm{CO}_{2}$ and

Table 3 Mechanical properties of PAES membranes

\begin{tabular}{llllr}
\hline Membrane & Thickness $(\mu \mathrm{m})$ & Tensile strength $(\mathrm{MPa})$ & Tensile modulus $(\mathrm{GPa})$ & Maximum elongation $(\%)$ \\
\hline Am-PAES-20 & 89 & 79 & 3.2 & 10.6 \\
Am-PAES-60 & 92 & 88 & 2.8 & 10.8 \\
Cl-PAES-20 & 90 & 75 & 3.3 & 9.6 \\
Cl-PAES-60 & 85 & 73 & 3.4 & 9.3 \\
Br-PAES-20 & 88 & 69 & 2.9 & 9.8 \\
Br-PAES-60 & 87 & 71 & 2.5 & 8.2 \\
PEP-PAES-20 & 90 & 78 & 3.2 & 8.8 \\
PEP-PAES-60 & 95 & 72 & 2.7 & 7.9 \\
TPEP-PAES-20 & 92 & 102 & 4.8 & 3.5 \\
TPEP-PAES-60 & 88 & 110 & 5.2 & 2.8
\end{tabular}


Table 4 Gas permeabilities of membranes

\begin{tabular}{|c|c|c|c|c|c|c|c|c|}
\hline \multirow[b]{2}{*}{ Membrane } & \multicolumn{4}{|c|}{ Permeability (barrer ${ }^{a}$ ) } & \multicolumn{3}{|c|}{ Ideal selectivity $^{b}$} & \multirow[b]{2}{*}{ FFV } \\
\hline & $\mathrm{O}_{2}$ & $\mathrm{~N}_{2}$ & $\mathrm{CO}_{2}$ & $\mathrm{He}$ & $\mathrm{CO}_{2} / \mathrm{O}_{2}$ & $\mathrm{CO}_{2} / \mathrm{N}_{2}$ & $\mathrm{O}_{2} / \mathrm{N}_{2}$ & \\
\hline Am-PAES-20 & 2.57 & 0.33 & 5.80 & 13.68 & 2.26 & 17.58 & 7.79 & 1.21 \\
\hline Am-PAES-60 & 2.14 & 0.22 & 6.78 & 12.88 & 3.17 & 30.82 & 9.72 & 1.17 \\
\hline Cl-PAES-20 & 3.22 & 0.45 & 6.26 & 18.45 & 1.94 & 13.91 & 7.16 & 1.28 \\
\hline Cl-PAES-60 & 3.65 & 0.50 & 6.82 & 25.75 & 1.87 & 13.64 & 7.30 & 1.35 \\
\hline Br-PAES-20 & 4.26 & 0.48 & 10.33 & 26.37 & 2.42 & 21.52 & 8.88 & 1.45 \\
\hline Br-PAES-60 & 3.72 & 0.43 & 8.78 & 25.66 & 2.36 & 20.42 & 8.65 & 1.57 \\
\hline PEP-PAES-20 & 5.73 & 0.57 & 10.44 & 32.78 & 1.82 & 18.32 & 10.05 & 1.54 \\
\hline PEP-PAES-60 & 8.66 & 0.64 & 13.56 & 38.15 & 1.57 & 21.19 & 13.53 & 1.62 \\
\hline
\end{tabular}

He permeabilities because of strongly hydrogen bond interaction between chains caused by $-\mathrm{NH}_{2}$ groups. This hydrogen bond interaction resulted in a decrease in FFV. It could be seen from Table 4, the gas permeability of anhydride modified PAESs increased with increasing of FFV of polymers. For instance, the polymer PEP-PAES-60 showed about times higher permeabilities than homologous precursor polymer. It was owing to the presence of the bulky phenylacetylene phthalic imide group in polymer pendant which hindered the compact packing of polymer chains.

The diffusion coefficient $(D)$ of gas molecules in membranes was obtained by the time-lag method, and the results were listed in Table 5. It is obvious that the increase in FFV would lead directly to an increase in the diffusivity of polymers, except $\mathrm{Br}$ PAES-60. Therefore, the permeability also increases with FFV while there is no obvious interaction between gas molecules and polymers. However, diffusion coefficients are higher for $\mathrm{Br}$ PEAS-20, even if the FFV is higher for Br-PEAS-60. Consequently the diffusion behavior is not only determined by difference in theoretical FFV value. The possible reason explained by Álvarez coworkers $^{28}$ is that the difference in permeability of Br-PEAS is mainly due to the difference in size distribution of free volume elements. In the case of Br-PEAS-20, the much higher permeability could be explained by a larger average size of free volume elements. The additional introduction of 4-bromonaphthalimide groups in Br-PEAS- 60 brought about a larger number of narrower free volume elements and obstructed the permeation of gas molecules, even though led to higher FFV.

For gas separation membranes, a trade-off generally exists between permeability and permselectivity and results in any

Table 5 Gas diffusivity $\left(10-8 \mathrm{~cm}^{2} \mathrm{~s}^{-1}\right)$ of membranes

\begin{tabular}{lrlll}
\hline Membrane & $D\left(\mathrm{O}_{2}\right)$ & $D\left(\mathrm{~N}_{2}\right)$ & $D\left(\mathrm{CO}_{2}\right)$ & $D(\mathrm{He})$ \\
\hline Am-PAES-20 & 3.41 & 0.64 & 2.45 & 17.35 \\
Am-PAES-60 & 2.56 & 0.36 & 2.04 & 12.54 \\
Cl-PAES-20 & 5.36 & 0.78 & 2.64 & 25.33 \\
Cl-PAES-60 & 7.86 & 0.98 & 3.35 & 28.36 \\
Br-PAES-20 & 10.55 & 1.88 & 3.65 & 37.44 \\
Br-PAES-60 & 8.74 & 1.45 & 3.12 & 32.02 \\
PEP-PAES-20 & 12.36 & 0.75 & 3.89 & 42.32 \\
PEP-PAES-60 & 15.52 & 0.85 & 4.42 & 48.12
\end{tabular}

improvement in permeability accompanied with a decrease permselectivity, and vice versa. As can be seen from Table 4, the results of separating property of anhydride modified PAESs almost followed the above rule, except for Br-PAES-20. Compared with Am-PAES-20, the $P\left(\mathrm{O}_{2}\right), P\left(\mathrm{~N}_{2}\right)$ and $P\left(\mathrm{CO}_{2}\right)$ of Br-PAES-20 increased from 2.57, 0.33 and 5.80 to $4.26,0.48$ and 10.33 barrers, respectively, accompanying with the increase in separation coefficients for three gas pairs.

\section{Conclusions}

Three kinds of imide group pendant were successfully introduced into poly(arylene ether sulfone). All modified polymers displayed good solubility in polar solvents, high thermal stability, and good mechanical properties. Gas transport properties for $\mathrm{O}_{2}, \mathrm{~N}_{2}, \mathrm{CO}_{2}$ and $\mathrm{He}$ had been investigated. Introduction of bulky imide moieties increased the FFV of polymers, thus resulted in increasing of gas permeability. In comparison with Am-PAES membranes, modified polymer membranes showed higher gas permeabilities. The gas permeabilities indicated that the introduction of imide groups into polymer pendant is highly effective to improve the gas permeability of membranes. Meanwhile, the permselectivity of modified PAES almost decreased due to the trade-off relationship between permeability and permselectivity. The only exception, Br-PAES20 , possesses higher permeability and permselectivity simultaneously. This anhydride modification approach may act as a simple and feasible option to improve gas separation performance of polymer membranes.

\section{Conflicts of interest}

There are no conflicts to declare.

\section{Acknowledgements}

The authors acknowledge Education Department of Liaoning Province for financial support for this work (Project No.: LJZ2016002 and L2015411). Moreover, National Natural Science Foundation of China (Project No.: 61503258) and Natural Science Foundation of Liaoning Province (Project No.: 201602614) also provided financial support for this work.

\section{References}

1 D. S. Sholl and R. P. Lively, Nature, 2016, 532, 435.

2 P. Bernardo, E. Drioli and G. Golemme, Ind. Eng. Chem. Res., 2009, 48, 4638.

3 D. F. Sanders, Z. P. Smith, R. Guo, L. M. Robeson, J. E. Mcgrath and D. R. Paul, Polymer, 2013, 54, 4729.

4 R. W. Baker, Membr. Technol., 2001, 138, 5.

5 Y. Yampolskii, Macromolecules, 2012, 45, 3298.

6 L. M. Robeson, J. Membr. Sci., 1991, 62, 165.

7 L. M. Robeson, J. Membr. Sci., 2008, 320, 390.

8 P. Pandey and R. S. Chauhan, Prog. Polym. Sci., 2001, 26, 853.

9 L. R. Burns and W. J. Koros, J. Membr. Sci., 2003, 211, 299.

10 S. K. Sen and S. Banerjee, J. Membr. Sci., 2010, 350, 53. 
11 C. E. Powell and G. G. Qiao, J. Membr. Sci., 2006, 279, 1.

12 M. T. Bessonov, M. M. Koton, V. V. Kudryavtsev and L. A. Laius, Polyimides: Thermally Stable Polymers, Consultants Bureau, New York, 1987.

13 D. Wilson, H. D. Stenzenberger and P. M. Hergenrother, Polyimides, Springer, Netherlands, 1990.

14 M. K. Ghosh and K. L. Mittal, Polyimides: Fundamentals and Applications, Marcel Dekker, New York, 1996.

15 M. M. Guo, B. J. Liu, L. Li, C. Liu, L. F. Wang and Z. H. Jiang, J. Power Sources, 2010, 195, 11.

16 Y. W. Yu, B. J. Liu, Y. Wang, S. Y. Liu, X. B. Li, Z. Liu and Z. H. Jiang, High Perform. Polym., 2014, 26, 401.

17 J. C. Jansen and E. Drioli, Polym. Sci., Ser. A, 2009, 51, 1355.

18 L. M. Robeson, Curr. Opin. Solid State Mater. Sci., 1999, 4, 549.

19 G. Maier, M. Wolf, M. Bleha and Z. Pientka, J. Membr. Sci., 1998, 143, 115.

20 J. Y. Park and D. R. Paul, J. Membr. Sci., 1997, 125, 23.
21 M. X. Ding, Polyimides: Condensation. Wiley Encyclopedia of Composites, John Wiley \& Sons, Inc, 2012.

22 X. Ma, Z. Lv, D. Wang, S. Guan, C. Chen, G. Wang, D. Zhang and Z. Jiang, J. Photochem. Photobiol., A, 2007, 188, 43.

23 B. J. Liu, G. B. Wang, W. Hu, Y. H. Jin, C. H. Chen and Z. H. Jiang, J. Polym. Sci., Part A: Polym. Chem., 2002, 40, 3392.

24 B. J. Liu, Y. Dai, G. P. Robertson, M. D. Guiver, W. Hu and Z. H. Jiang, Polymer, 2005, 46, 11279.

25 G. Chen, X. S. Zhang, S. B. Zhang, T. L. Chen and Y. L. Wu, J. Appl. Polym. Sci., 2007, 106, 2808.

26 W. J. Koros, G. K. Fleming, S. M. Jordan, T. H. Kim and H. H. Hoehn, Prog. Polym. Sci., 1988, 13, 339.

27 Z. G. Wang, T. L. Chen and J. P. Xu, Macromolecules, 2001, 34, 9015.

28 M. Calle, C. García, A. E. Lozano, G. J. de la Campa, J. de Abajo and C. Álvarez, J. Membr. Sci., 2013, 434, 121. 